
\title{
Divero 'Sidade
}

LIGAÇÕES E COMUNIDADE: INTRODUÇ̃̃O DA ÉTICA DO CUIDADO NA EDUCAÇÃO ATRAVÉS DE ESTRUTURAS PARTICIPATIVAS.

\section{VÍNCULOS Y COMUNIDAD: INTRODUCCIÓN DE LA ÉTICA DEL CUIDADO EN EDUCACIÓN A TRAVÉS DE ESTRUCTURAS PARTICIPATIVAS.}

\section{EMOTIONAL BONDS AND COMMUNITY: INTRODUCTION OF THE ETHICS OF CARE IN EDUCATION THROUGH PARTICIPATORY STRUCTURES.}

\author{
Alicia Bernardos Hernánde ${ }^{1}$ \\ Irene Martínez-Martín ${ }^{2}$ \\ Irene Solbes Canales ${ }^{3}$
}

\section{RESUMO}

Este artigo descreve o projeto desenvolvido desde 2017 numa escola secundária pública da Comunidade de Madrid. O objetivo do projeto é introduzir gradualmente a ética do cuidado na organização do centro. Presta atenção ao desenvolvimento da identidade relacional dos alunos, promove laços de apoio e ajuda e promove ações para a melhoria dos bens comuns. Por meio de uma pesquisa-ação desenvolvida a partir de um seminário de formação de professores, foram construídas estruturas participativas em todos os níveis do ensino médio obrigatório, que envolveram espaços de escuta de necessidades, e permitiram: a articulação dos alunos com interesses compartilhados, o desenvolvimento de relações de cuidado, o envolvimento na melhoria da instituição e a resolução de conflitos de forma dialogada. Conclui-se que esta linha de ação aumenta o bem-estar coletivo, objetivo último da educação.

PALAVRAS-CHAVE: Ética do cuidado. Identidade relacional. Pesquisa-ação.

Pedagogia feminista.

\section{RESUMEN}

El presente artículo describe el proyecto desarrollado durante los cursos 2017/18 y 2018/19 en un instituto público de educación secundaria de la Comunidad de Madrid (España). El objetivo fue introducir paulatinamente la ética del cuidado en la organización del centro, prestando atención al desarrollo de la identidad relacional del alumnado, y fomentando tanto vínculos de apoyo y ayuda, como acciones para la mejora de lo común. A través de una investigación - acción desarrollada a partir de un

\footnotetext{
${ }^{1}$ Master en Calidad y Mejora en Educación. Universidad Complutense, Madrid, España.

${ }^{2}$ Doctora en Educación. Universidad Complutense, Madrid, España.

${ }^{3}$ Doctora en Psicología. Universidad Complutense, Madrid, España.
} 
seminario de formación del profesorado, se construyeron estructuras participativas en todos los niveles de enseñanza secundaria obligatoria, que supusieron espacios de escucha de necesidades, y que permitieron: la vinculación del alumnado con intereses compartidos, el desarrollo de relaciones de cuidado, la implicación en la mejora de la institución, y la resolución de conflictos de forma dialogada. Se concluyó que esta línea de acción aumenta el bienestar colectivo, fin último de la educación.

PALABRAS-CLAVE: Ética del cuidado. Identidad relacional. Investigación-acción.

Pedagogía feminista.

\section{ABSTRACT}

This article describes a project developed in a public school in Madrid since 2017. The aim of the project is to introduce gradually the ethics of care into the organisation of the centre. The project focuses on student's relational identity development. It also aims to foster bonding of support and help, and promotes actions that achieve the common goods. Through an action research methodology, developed from a teacher training seminar, participative structures were built at all levels of compulsory secondary education, which involved the creation of listening spaces where needs are heard. Those structures allowed emotional bonds between students with shared interests, the development of relationship-centered care, involvement in the institution's improvements, and conflict resolution through dialogue. It therefore follows that this line of action enhances collective well-being, the ultimate goal of education.

KEYWORDS: Ethics of care. Relational identity. Action-research. Feminist pedagogy.

\section{Introducción \\ Introduccion}

$$
* * *
$$

Este artículo recoge la experiencia del proyecto "Comunidad de Cuidados", tal y como se desarrolló durante los cursos 2017 - 18 y 2018 - 19 en el IES MP, instituto público de educación secundaria situado en la periferia sur de Madrid (España). El objetivo del proyecto fue introducir paulatinamente la ética del cuidado en la organización de la institución, prestando atención al desarrollo de la identidad relacional del alumnado y fomentando vínculos de apoyo, ayuda y de trabajo en común que promuevan el bienestar de las personas que conviven en la escuela.

El proyecto se fundamenta desde un marco teórico de pedagogía crítica y feminista, alternativa al modelo educativo que se promueve actualmente en nuestra Comunidad Autónoma. Este modelo asume un concepto de escuela como espacio de desarrollo de competencias individuales, una "fábrica de rendición de cuentas", aplicándose los planteamientos propios de la economía neoliberal e imitando la lógica instrumental del mercado (GIROUX, 2019). Para Gimeno Sacristán (2019), la orientación economicista es "agresiva", y afecta tanto al discurso, como a la organización, el currículum y las prácticas de aula. 
La consecuencia del enfoque de rendición de cuentas es la priorización de los objetivos de logro, las fórmulas sofisticadas de evaluación dirigidas a aquello que puede ser medido, y la competición entre centros (GIMENO-SACRISTÁN, 2018). Este modelo promueve una educación basada en contenidos o habilidades, con gran atención a las capacidades técnicas del profesorado vinculadas al dominio de la materia a enseñar, a la programación y evaluación medible de contenidos (ÁLVAREZ, 2015). Las pruebas de evaluación externas magnifican el enfoque en el logro de objetivos unificados, y minimizan los efectos de experiencias plurales y diversas en la construcción de identidades y saberes (SKELTON y FRANCIS, 2009).

Según la UNESCO (2015), es necesario superar la visión utilitaria y de capital humano que caracteriza el discurso educativo del desarrollo, puesto que "se puede afirmar que mantener y aumentar la dignidad, la capacidad y el bienestar de la persona humana en relación con los demás y con la naturaleza, debería ser la finalidad fundamental de la educación en el siglo XXI" (p. 36). Si la cuestión central de la educación tiene que ver con la dignidad y el bienestar de la persona en relación con otros seres humanos y no humanos, esta cuestión nos lleva a interrogarnos sobre cuáles son las condiciones que permiten que una vida merezca la pena ser vivida (HERRERO, 2019), y cómo contribuir desde lo educativo a construir estas condiciones. Tal y como lo enuncia Marina Garcés:

Hay una pregunta, sin embargo, que ninguna forma de dogmatismo solucionista podrá llegar nunca a resolver. Es la pregunta que La Boétie, en el siglo XVI, consideraba la raíz de toda insubordinación a la servidumbre voluntaria. ¿Es esto vivir? (GARCÉS, 2017, p.58).

Por todo ello, volvemos la mirada hacia las pedagogías críticas y feministas para profundizar en el planteamiento de la ética del cuidado, enfoque capaz de orientar un cambio de modelo: desde de un planteamiento donde prima la autonomía individual, el desarrollo de aptitudes orientadas al futuro y la productividad, a uno que pone en el centro la vida humana entendiéndola como vulnerable e interdependiente, y que se apropia de la posibilidad de transformación del presente, "del tiempo vivible y de sus condiciones compartidas, recíprocas e igualitarias” (GARCÉS, 2017, p. 74).

\section{Marco teórico:}


En 1982, Carol Gilligan publicó In a Different Voice, poniendo en cuestión los resultados descritos por Kohlberg (1971) sobre las fases de desarrollo del razonamiento moral. Kohlberg describió cómo los hombres alcanzaban con mayor facilidad el nivel que era considerado superior: la capacidad de aplicación de normas abstractas universales a dilemas éticos complejos. Gilligan observó entonces una voz diferente en la forma que tenían algunas niñas de enfrentarse a estos dilemas, más vinculada a las situaciones concretas y a las relaciones implicadas. Esta voz diferente, denominada "ética del cuidado", fue desarrollada posteriormente a nivel teórico por Nel Noddings (1992) y una línea de teóricas feministas, mostrándose como una alternativa a otras aproximaciones morales tradicionales: en lugar de apelar a normas abstractas, aprovecha el potencial de la experiencia personal de cuidado e interdependencia por la que todo ser humano ha pasado en su vida (HELD, 2005).

El concepto de "cuidado" ha incluido tres aspectos en este desarrollo: es un tipo de ética (que se describe en contraposición con la ética de la justicia); es una forma de relación; y es un conjunto de trabajos -y no se debe perder la visión de quién hace mayoritariamente estos trabajos y en qué condiciones- (RUDDICK, 1998).

El feminismo considera que el cuidado como ética debe ser reconocido, y no asumido como una inclinación de la naturaleza femenina, para la que no es necesario aprendizaje (GILLIGAN, 1982; NODDINGS, 1992). Denominada también "ética relacional", desplaza el interés desde lo individual a la interacción, y sus aplicaciones van más allá de los aspectos privados: es aplicable a ámbitos públicos e institucionales, convirtiéndose en acción política. El elemento central de la ética del cuidado, o relacional, es el hecho de que las decisiones morales no se basan en el interés individual, sino en intereses entrelazados en situaciones concretas, en las que se tiene en cuenta el bien común y la relación de interdependencia, proponiendo acciones situadas más que razonamientos abstractos y neutros. Los aspectos emocionales no sólo no se dejan de lado, sino que se consideran centrales para la toma de decisiones (HELD, 2005; NODDING, 2013).

El cuidado como relación describe interacciones basadas en la receptividad, la sensibilidad, la interdependencia y la respuesta a las necesidades en situaciones específicas. Aunque existen disposiciones personales que facilitan las relaciones de cuidado, éstas no las garantizan. Las características son inherentes a la relación, y no podrán asumirse a partir de la suma de las disposiciones de las personas implicadas: el 
vínculo de dos personas receptivas hacia las necesidades de la otra no determina que establezcan entre ellas una relación de cuidado (HELD, 2005).

El cuidado como trabajo, según Tronto y Fisher (1990, p. 40) alude a aquellas actividades de la especie que mantienen y repararan nuestro mundo, para que podamos vivir en él lo mejor posible, construyendo una red que sostiene la vida.

El cuidado es un término muy amplio que se refiere a todas las actividades destinadas a proporcionar bienestar físico, psíquico y emocional a las personas. Todo el trabajo imprescindible para el mantenimiento y reproducción de la vida, y otros cuidados necesarios para nuestra calidad de vida. Abarca el trabajo doméstico, que siempre hemos realizado las mujeres en el espacio privado, lo que explica por qué los cuidados son prestados mayoritariamente por las mujeres, y por qué está naturalizado que seamos las mujeres quienes los realicemos. (CÁRDABA, 2013, P. 27).

Supone por tanto un sistema de vínculos y tareas que permiten la supervivencia y el bienestar de cuerpos vulnerables y dependientes desde el nacimiento, con un fuerte componente patriarcal en el reparto desigual de las cargas que implica.

Hernando (2012) ha argumentado cómo una característica de las sociedades patriarcales es la invisibilidad de esta interdependiencia entre las personas, y la devaluación en el discurso social de los vínculos y las emociones. La idea de la autonomía individual se construye sobre la falsedad de que la vida sin dependencias es posible, siendo un eje central del proceso civilizatorio occidental la ficción de poder vivir emancipados de la naturaleza, de nuestro propio cuerpo o del resto de las personas (HERRERO, 2018).

En los distintos usos del término cuidado descritos, se parte de un concepto de identidad que no es previa a las relaciones, sino constituida por ellas, desde los vínculos de reciprocidad establecidos en los grupos de pertenencia. La autonomía personal es, en este contexto, distinta de la idea de autosuficiencia, no interferencia, autodirección, control racional, etc., propia del modelo neoliberal (HELD, 2005). La consideración de la identidad como eminentemente relacional implica que el interés por el bienestar ajeno no sea independiente del interés por el propio bienestar. Como recuerda Lawrence Blum (citado por HELD, 2005, p. 35), preocuparse por una amiga o por una comunidad de la 
que se es miembro, no es acercarse a alguien o a algo totalmente distinto de una misma, sino a algo con lo que se comparte una parte del yo, implicado en el sentido de la propia identidad.

\section{Política de cuidados en la institución educativa}

La línea pedagógica feminista defiende un cambio de modelo que vaya más allá de las demandas de igualdad de oportunidades entre hombres y mujeres (MACEIRA, 2006), proponiendo una ruptura de las normas hegemónicas patriarcales que se reproducen en el contexto educativo (MARTÍNEZ-MARTÍN, 2016; MARTÍNEZMARTÍN, 2018; MARTÍNEZ-MARTÍN y RAMÍREZ-ARTIAGA, 2017). Desde este marco, la perspectiva de la ética del cuidado puede ser aplicada a las instituciones educativas, lo que plantea problematizar cada aspecto de la escolarización: el modelo de organización del tiempo, los espacios y la arquitectura, el tamaño de las escuelas y las clases, el tipo de relaciones que se alientan, la estructura jerárquica, las categorías lingüísticas que se aplican, los objetivos de la enseñanza, la selección de contenidos, las formas de evaluación, etc. (NODDINGS, 2013).

Es necesario entender la institución como un sistema, un organismo cambiante formado por complejas interacciones. La escucha en la escuela deberá ser atenta y abierta, y no será posible extrapolar soluciones de un lugar a otro de forma acrítica:

Escuchar -en- la escuela implica quedarse sorda. "Desoír" las retóricas y los lenguajes descarnados que permanentemente la enuncian para no decir nada. Sordas al habla abstracta, escuchas atentas de su ritmo, de lo sensible, de su ambigua vitalidad. (...) Escuchar la escuela, entonces, para habitarla de otro modo, no para interpretarla o intervenirla: escucharla para experimentarla.

(ESCRITURA DE PARACAIDISTA, 2016, p.4).

Partiendo de estas premisas, una escuela que asume una política de cuidados tendrá en cuenta aspectos como los siguientes:

- Se explicitarán y se convertirán en centrales aquellas tareas que implican la atención a necesidades, en toda la amplitud del concepto. Puesto que en las instituciones educativas conviven diferentes necesidades, a menudo contradictorias y cambiantes, es imprescindible reservar tiempos y espacios claros para la gestión de conflictos complejos a través del diálogo. Las 
necesidades tienen que ser comprendidas, escuchadas, negociadas, priorizadas... Las mejores formas de cuidado son aquellas que son altamente deliberadas y explícitas, lo que requiere que la escuela construya espacios adecuados para dialogar y articular el cuidado a todos sus miembros, un proceso dinámico que no tiene punto de llegada (TRONTO, 2010).

- Se pondrá el foco en el tipo de relaciones que se promueven, y no tanto en las disposiciones personales. Se fomentarán las relaciones que ayudan a sostener el bienestar entre estudiantes, favoreciendo las interacciones entre iguales, y valorando la calidad de estas relaciones tanto como los resultados académicos.

- Se promoverá la motivación de las personas para involucrarse en relaciones de cuidado dentro y fuera de la institución, especialmente entre los alumnos y las alumnas, fomentando disposiciones como: la sensibilidad para percibir las necesidades propias y ajenas, la confianza en la relación, el interés por la cooperación (y el rechazo a obtener ventaja personal de las situaciones de interdependencia), y el autocuidado. En definitiva, se potenciará la habilidad para escuchar necesidades y la responsabilidad para responderlas, haciendo hincapié en estos aspectos en la socialización de los chicos. Es necesario compensar las desigualdades en la socialización diferenciada de los sexos, que se mantiene de una forma poco visible y naturalizada (SUBIRATS, 2016), potenciando la capacidad masculina de cuidado.

- Se cultivarán las relaciones de cuidado dentro del aula. Resaltamos tres estrategias fundamentales derivadas del trabajo de Nel Noddings (BROOME, BOBICK, RUGGIERO y JESUP, 2019): (a) ofrecer oportunidades para un diálogo que corrija y enriquezca las perspectivas individuales, teniendo en cuenta las desigualdades de poder; (b) diseñar trabajo cooperativo que permita al alumnado compartir responsabilidades en la construcción de un saber común; y (c) un modelado constante de comportamientos de cuidado por parte de la/el docente.

- Se incidirá en la interdependencia humana, haciendo visible la importancia de los vínculos para la supervivencia y el bienestar, del trabajo colectivo para la consecución de objetivos, y cuestionando la idea de triunfo individual. Para ello, además de abordar estas cuestiones a nivel curricular, es importante tejer poder comunitario, promoviendo en el centro el desarrollo de una identidad 
colectiva y la participación real de la comunidad educativa (HERRERO, 2019).

- Se adoptará una perspectiva crítica ante la distribución de las cargas de trabajo de cuidados en los contextos próximos al alumnado. Held (2005) advierte que una ética del cuidado que lo ensalza, pero no se preocupa de cómo se distribuyen sus cargas, contribuye a la explotación de las mujeres y de las personas que realizan el trabajo de atención no remunerado o en condiciones precarias. El análisis y evaluación de cómo los cuidados han evolucionado en las condiciones históricas de dominación patriarcal puede permitir imaginar nuevas formas de organización en el futuro.

- Se alentará la diversidad, con una política antinormativa en el lenguaje cuando se emplea en la categorización de las personas, en las descripciones en informes y juntas de evaluación. El objetivo es no patologizar la diversidad ni ensalzar la normalidad, siendo capaces de incluir enfoques interseccionales (SÁNCHEZ-SÁINZ, 2019).

Por lo tanto, una educación que sitúa en el centro los cuidados debe favorecer las relaciones de apoyo mutuo, construir marcos centrados en la ética de la reciprocidad, estimular en la socialización de jóvenes la cultura de "hacerse cargo" y la preocupación por el bien común como base de un compromiso ético, con un cuestionamiento del modelo de_desarrollo dominante y su distribución de las tareas de cuidado.

La Pedagogía del Cuidado está llena de potencial por desarrollar y puede dar respuesta a muchos de los interrogantes que en estos momentos se plantea la Educación, como son los relativos a su sentido profundo, al papel que juega en la actual coyuntura histórica y a cómo motivar a un profesorado y un alumnado que en muchas ocasiones se siente obligado a participar en un espacio educativo en el que no cree (AGUADO, 2018).

Recogiendo estas propuestas, en el IES MP se ha priorizado el tejido de estructuras organizativas que promueven el encuentro y vinculación de personas, y el establecimiento de relaciones de cuidado en el centro, como desarrollamos a continuación. 


\section{Diseño de la investigación acción y objetivos.}

El instituto MP, ubicado en la periferia sur de Madrid, desarrolla desde el año 2017 un proyecto denominado Comunidad de Cuidados, promoviéndose actuaciones que han llevado a alumnas y alumnos a realizar actividades en el centro que van más allá de recibir clase, interiorizar contenidos o desarrollar habilidades concretas útiles para el futuro.

El proyecto Comunidad de Cuidados constituye una experiencia de investigaciónacción participativa continua, que surge del deseo de construir un instituto más vivible, y que es dinamizada por un grupo de profesores y profesoras constituidos en seminario de formación, junto con el departamento de orientación y el nuevo equipo directivo del centro. La investigación - acción supone un proceso de reflexión en la acción, un proceso cíclico de acción-análisis-reflexión-acción (BOTELLA y RAMOS, 2019).

Describimos a continuación el proyecto desarrollado durante los cursos 2017 - 18 y 2018 - 19, que consistió en implementar distintas estructuras organizativas en el centro y evaluar su efectividad en la mejora de las relaciones de apoyo, los vínculos afectivos y la experiencia de bienestar en la institución. El objetivo que supuso el punto de partida del proceso fue promover, a través de propuestas organizativas del centro, grupos de afinidad y relaciones de cuidado, favoreciendo los aspectos relacionales en la construcción de la identidad adolescente, y dando visibilidad y relevancia a las vivencias emocionales. Considerábamos que las acciones diseñadas a partir de este objetivo mejorarían la sensación de bienestar subjetivo de las personas que conviven en la escuela.

Más concretamente, nos propusimos los siguientes objetivos:

- Articular en la organización del centro espacios de escucha y negociación de toda la comunidad educativa, prestando especial atención al alumnado.

- Reconocer, dar visibilidad y potenciar las relaciones de cuidado entre el alumnado.

- Promover redes de apoyo basadas en intereses comunes, que supongan un sostén emocional, especialmente a aquellas alumnas y alumnos más vulnerables, o en una situación de menor privilegio.

- Promover el diálogo entre alumnas y alumnos en situación de conflicto, fomentando la capacidad de escucha, así como la disposición a enriquecer la perspectiva individual. 
- Potenciar el sentido de centro como bien común, promoviendo las acciones que incidan en su transformación hacia un espacio de bienestar, especialmente para el alumnado.

\section{Acciones: estructuras participativas, ayudantes y mediación.}

Este planteamiento general se desarrolló gracias a la iniciativa del nuevo equipo directivo y con la ayuda de un seminario de formación permanente para el profesorado desarrollado en el instituto y denominado "Comunidad de Cuidados". El proyecto incluye tres líneas de trabajo -estructuras participativas, ayudantes y equipo de mediación- que se describen a continuación.

La primera línea, dirigida a $1^{\circ}$ y $2^{\circ}$ de ESO, denominada ESTRUCTURAS PARTICIPATIVAS, se desarrolló en la hora de tutoría -un período semanal incluido en el horario de permanencia con la tutora o el tutor. En estos dos niveles la tutoría se hizo coincidir en el horario, lo que nos ofreció una hora semanal en la que todo el alumnado de cada uno de los dos niveles no estaba recibiendo docencia de una materia curricular. La primera tutoría de cada mes se rompió el grupo clase, permitiendo a todo el alumnado acudir a la estructura participativa que había elegido a principio de curso.

Las temáticas de las estructuras que se ofrecieron al alumnado tenían relación con dos aspectos:

- actividades que podían redundar en el bienestar de compañeras y compañeros en el centro (por ejemplo, Representantes de grupo, Ecoescuela, o Agentes de convivencia).

- creación de grupos de afinidad, algunos especialmente diseñados para alumnado vulnerable (por ejemplo, el taller de Comic y fanzine, o Agentes de diversidad).

A principio de curso el alumnado eligió aquel tema que respondía a sus intereses, partiendo de un listado de once posibilidades, entre las que estaban, además de los nombrados anteriormente: Agentes de salud, Premonitores de tiempo libre, Ocio saludable, Taller de películas, Lengua de signos, etc. La oferta de temáticas permitió ratios bajas (entre 10 y 20 estudiantes por grupo), y las sesiones fueron impartidas por distintos agentes y asociaciones de la comunidad, principalmente de recursos de ocio, sociales, educativos, y de salud comunitaria del barrio. Por tanto, una vez al mes, y con el alumnado interesado, se trabajaron aspectos que redundaron en la mejora del centro, 
permitiendo así la creación de lazos entre personas con intereses afines, y formando al alumnado en la responsabilidad respecto al bienestar colectivo.

La segunda línea, dirigida a $3^{\circ}$ y $4^{\circ}$ de ESO, se denominó AYUDANTES, y ofreció al alumnado la posibilidad de participar en una estructura de cuidados y mejora de la institución a lo largo de todo el curso. En el curso 2018 - 19 esta estructura estuvo formada por un grupo de 35 estudiantes voluntarias y voluntarios de $3^{\circ}$ de ESO. El grupo funcionó como una pequeña comunidad dentro del instituto, realizándose asambleas con la orientadora en horario de recreo desde el principio hasta el final de curso. La asamblea se convirtió en un espacio de participación directo, en el que la orientadora del instituto acompañó las dinámicas que surgían del grupo, fomentándose un estilo de comunicación horizontal. Las acciones del grupo se decidieron en cierta igualdad, tratando de reducir la influencia de la opinión adulta y potenciando la importancia de la opinión adolescente, para así equilibrar la desigualdad de poder. La asamblea del grupo de ayudantes supuso por tanto un espacio de escucha del alumnado, un lugar de libertad para expresar sus opiniones, deseos e iniciativas, y una fórmula de desarrollo directo de sus ideas. En ella se planificaron acciones como las siguientes:

- Jornadas de acogida para el alumnado que se incorporaba desde los colegios, encargándose el alumnado de la recepción, visita al centro y juegos de conocimiento.

- Actividades de tutoría para el alumnado de primero de ESO, relacionadas con la convivencia, la resolución de conflictos de forma no violenta, y la diversidad entendida como riqueza, entre otros temas. Las actividades de tutoría se desarrollaron en forma de grupos interactivos, con alumnado ayudante situado en cada pequeño grupo, proponiendo cuestiones para la reflexión y recogiendo opiniones. No supusieron por tanto charlas, sino sesiones dinámicas en las que cinco alumnas y alumnos de tercero se distribuían en pequeños grupos en cada clase de primero, favoreciendo la participación de todo el grupo con textos y preguntas. Esta metodología permitió una experiencia de diálogo real con el alumnado más joven a cerca de temáticas con escaso tratamiento en la escuela, como la diversidad afectivo-sexual.

- Presentación del centro a las familias en las jornadas de puertas abiertas, realizando la visita guiada y la explicación de proyectos de participación del alumnado. 
- Acciones en torno al 8 de marzo: publicación de un fanzine elaborado por las propias alumnas, exposición de punto feminista en la biblioteca, y acción directa de difusión el 7 de marzo en el recreo, diseñada y realizada por el alumnado.

Por último, se constituyó el EQUIPO DE MEDIACIÓN, formado por alumnas y alumnos de tercero de ESO y dos profesoras, además de la implicación del equipo directivo para la derivación de conflictos. Desde el centro se realizó una formación específica para llevar a cabo mediaciones informales y formales, favoreciendo una cultura de resolución dialógica de conflictos entre adolescentes y favoreciendo el buen trato. La formación y la realización de mediaciones se orientaron a favorecer la escucha y la comprensión empática de la otra parte, considerando como deseable -pero no imprescindible- el proponer una solución para el conflicto concreto que se trataba. Las y los adolescentes de $3^{\circ}$ y $4^{\circ}$ de Educación Secundaria Obligatoria guiaban al alumnado más joven en su narración del conflicto y en la comprensión de la situación por ambas partes, creando un clima en el que se suponía la mejor de las intenciones de las personas implicadas. La posibilidad de explicar, escuchar y ser escuchada/o, el reconocimiento de las emociones, y la devolución de información aclaratoria, suponía una experiencia única para las personas implicadas, en la que eran tenidas en cuenta más allá del hecho concreto que motivaba la petición de mediación (en muchas ocasiones una pelea). El grupo de mediación se reunía los viernes alternos para analizar los aspectos relacionales que alumnado y profesorado observaba en el instituto, suponiendo un lugar para la reflexión sobre las necesidades de las personas entendidas como seres relacionales.

\section{Evaluación del proceso.}

La evaluación de las acciones se realizó de forma continua, por el equipo directivo, en el seminario de formación Comunidad de Cuidados, y a través de la reflexión en cada actividad, asamblea, o reunión del grupo de mediación. Además, se aplicó un cuestionario para realizar una evaluación final de cada una de las líneas del proyecto, con el fin de conseguir una recogida más amplia de la opinión de las personas participantes.

En relación con las ESTRUCTURAS PARTICIPATIVAS de tutoría en $1^{\circ}$ y $2^{\circ}$ de ESO, las opiniones de las personas que tuvieron el papel de facilitadoras en los distintos grupos destacan como aspectos positivos: la riqueza de la ruptura del grupo clase; la 
oportunidad de trabajar temáticas no incluidas en el currículum académico, pero de suma importancia para el bienestar personal; y la posibilidad de que alumnas y alumnos con intereses comunes se vinculen. También se señaló la riqueza de permitir que distintas instituciones entraran en la escuela para desarrollar actividades de cierta duración y relevancia. Todas las personas encuestadas manifestaron su deseo de que estas actividades se mantuvieran, y de participar en las mismas. Por parte del alumnado participante, la puntuación relacionada con su satisfacción respecto a la participación en las estructuras participativas alcanzó un valor medio de 7’71 puntos (en una escala de 0 a 10). Por otro lado, ante la pregunta de si deseaban que las estructuras participativas se mantuvieran, la respuesta obtuvo una media de 8'44. Estas medias indican que, para la mayor parte del alumnado, la participación en estas estructuras fue satisfactoria o muy satisfactoria.

Por su parte, el grupo de AYUDANTES, en su autoevaluación, incidió en la sensación de bienestar que les proporcionó la implicación en un proyecto colectivo. Todas las actividades realizadas por el grupo fueron valoradas como altamente satisfactorias por parte del profesorado, las familias, y el alumnado participante. Destacamos la alta aprobación de las actividades de tutoría conducidas por el alumnado ayudante, consideradas las más satisfactorias de todas las programadas en la acción tutorial, por la posibilidad de diálogo real y respuesta a los intereses de los participantes de $1^{\circ}$ de ESO.

Existió una continuidad en el grupo: de las 35 personas que participaron al inicio, 29 continuaban en junio. En este curso, 26 personas del grupo de ayudantes de tercero han decidido continuar en el proyecto en cuarto de la ESO, ampliándose con cuatro personas nuevas. Su progresivo empoderamiento se comprobó en su iniciativa para hacer nuevas propuestas de acción; por ejemplo, la elaboración de carteles para la mejora de la autoestima junto a los espejos en los servicios de las chicas.

La observación cualitativa de la orientadora le lleva a apreciar el apoyo del equipo directivo a las acciones propuestas por el grupo de ayudantes, dirigidas al acompañamiento del alumnado más joven u otras. Esto permitió una experiencia de activismo exitoso del alumnado en la institución de la que formaban parte. Los momentos de diálogo asambleario con toma de decisiones, reparto de papeles y articulación de estrategias de difusión de un mensaje, supusieron episodios de acción colectiva, a nuestro juicio una vivencia necesaria y reveladora para el alumnado participante. Su papel fue reconocido por la institución con una beca en las actividades 
extraescolares durante todo el curso, y un diploma que se entregó en la fiesta de final de curso.

La MEDIACIÓN del propio alumnado para la resolución de conflictos fue valorada como una estrategia necesaria, aún en proceso de implementación. En el contexto en el que se encuentra el instituto, las amenazas y peleas físicas como forma de resolver conflictos son frecuentes. Consideramos como un primer paso en el establecimiento de una cultura de resolución de conflictos de forma dialogada algunas de las acciones acometidas: la constitución de un equipo de mediación, la difusión y la realización de mediaciones formales para la resolución de conflictos. Sin embargo, la consolidación de esta cultura, que lleve al alumnado a solicitar ayuda directamente al alumnado mediador, es un proceso que requerirá tiempo. Por ello, este curso profundizamos en la difusión y reconocimiento del proceso en toda la institución, con iniciativas propuestas por el propio grupo, como el uso de redes sociales para el contacto con el alumnado mediador.

En general, a través de diversas fuentes directas (propios estudiantes implicados) o indirectas (observaciones profesorado, redes sociales, familias...) se recaban evidencias de una mejora en la sensación de bienestar por parte de toda la comunidad educativa, a través de indicadores como los siguientes:

Las distintas entidades del barrio (servicios sociales, centro de salud, centro de la mujer...) manifiestan encontrar un clima en el centro que favorece el sentido de pertenencia de los colectivos más vulnerables, aconsejando nuestro centro en estas ocasiones.

- Las familias tienen un mayor deseo de participación en el AMPA, por lo que se ha aumentado significativamente el número de familias socias.

El profesorado repite en la elección de nuestro centro.

- $\quad$ El alumnado, en el test Sociescuela aplicado a todo el alumnado de la ESO en el curso 2018-19, ante la pregunta "¿cómo te encuentras en tu clase del instituto?" responde mayoritariamente entre "bien" y "muy bien", y en este aspecto nuestro centro supera la media de la Comunidad de Madrid en la comparativa que ofrece el propio test.

Este tipo de indicadores de bienestar, que no son valorados por las evaluaciones externas, sí son tenidos en cuenta por el equipo docente y directivo, haciéndonos considerar que se están construyendo las condiciones para que la comunidad educativa 
que convive a diario en esta institución se sienta acogida en su diversidad y escuchada en sus necesidades.

\section{Conclusiones}

Introducir como eje de decisión en la institución educativa la ética del cuidado supone considerar la atención a las necesidades y a los vínculos como aspectos prioritarios: entender que los seres humanos somos interdependientes y relacionales, estamos constituidos por nuestras relaciones y sobrevivimos gracias al cuidado mutuo. Nuestro bienestar depende de redes que responden a necesidades, y nuestros intereses se entrelazan con los de las personas con las que compartimos los espacios. Por tanto, la institución educativa debe dirigir la mirada al tipo de relaciones que promueve, educando identidades críticas hacia los logros individuales que invisibilizan la importancia de la interdependencia y rompiendo con los valores de competitividad e individualismo. Todas las personas escolarizadas tienen la vivencia de transitar a diario un espacio colectivo, lo que supone una valiosa oportunidad para la vivencia de vínculos de cuidado, el desarrollo de disposiciones de responsabilidad, de pertenencia al espacio próximo, de sensibilidad ante las necesidades propias y ajenas, y la acción para la mejora de lo común. Estos aspectos conectan con el fin de la educación para el siglo XXI declarado por la UNESCO en 2015: aumentar el bienestar colectivo.

La introducción de la ética del cuidado en el centro tiene implicaciones a múltiples niveles, desde la arquitectura de los centros a las ratios de los grupos, pasando por la formación y selección del profesorado, la selección de contenidos y metodologías o la elaboración de grupos y horarios, así como los modelos de relación, comunicación y resolución de conflictos. En nuestro centro, este modelo se introdujo a través de estructuras organizativas de participación y mejora de la institución por parte del propio alumnado, con resultados que nos animan a continuar moviéndonos en esta dirección. En este curso (2019-20) dando continuidad al proyecto, iniciamos la transformación de espacios desde esta perspectiva, de manera que paulatinamente se irán realizando cambios más ambiciosos para avanzar hacia un objetivo compartido: fomentar que las personas que conviven en nuestra institución se vivan como seres relacionales e interdependientes, y se impliquen en relaciones de cuidado y vínculos de apoyo, comprometiéndose con la mejora colectiva de lo común. 


\section{Referencias}

AGUADO, Guillermo y cols. Pedagogía de los cuidados: aportes para su construcción. Fundación InterRed, 2018. Disponível em

https://intered.org/pedagogiadeloscuidados/wp-content/uploads/2018/05/MarcoTeorico_Completo.pdf. Acesso em 6 nov. 2019.

ÁLVAREZ MÉNDEZ, Juan Manuel. La evaluación educativa en una perspectiva crítica: dilemas prácticos. Revista educación y cultura, v. 0000, n. 0111, p. 8-15, 2015.

BOTELLA, Ana María; RAMOS, Pablo. Investigación-acción y aprendizaje basado en proyectos. Una revisión bibliográfica. Perfiles educativos, v. 41, n. 163, p. 127-141, mar 2019. Disponível em

http://www.scielo.org.mx/scielo.php?script=sci_arttext\&pid=S0185-

$26982019000100127 \& \operatorname{lng}=$ es\&nrm=iso. Acesso em 28 oct. 2019.

BROOME, Jeffrey L.; BOBICK, Bryna; RUGGIERO, Alyssia; JESUP, Cindy. Responding to the Challenge to Care: Suggestions for Art Education Curricula. Art Education. v. 72, n. 2, p. 36-41, mar 2019. Disponível em https://doi.org/10.1080/00043125.2019.1559604. Acesso em 1 ago 2019.

CÁRDABA, Marta. El feminismo ante la privatización de los cuidados. En FORUM DE POLÍTICA FEMINISTA. Feminismo frente al neoliberalismo: estrategias y alianzas: XXIII Taller de Política Feminista, Madrid: Fórum de Política Feminista, p. 51-54, 2013. Disponível em http://www.forumpoliticafeminista.org/sites/default/files/Libro\%20XXIII\%20Taller\%2 094\%20pags\%20definitivo\%20web.pdf. Acesso em: 6 sep. 2019.

GARCÉS, Marina. Nueva ilustración radical. Barcelona: Editorial Anagrama, 2017. $75 \mathrm{p}$.

GILLIGAN, Carol. In A Different Voice: Psychological Theory and Women's Development. Cambridge: Harvard University Press, 1982. 220 p.

GIMENO-SACRISTÁN, José. Ya no necesitamos las ideas para legitimar las políticas. In: GIMENO-SACRISTÁN, José (Ed.). Cambiar los contenidos, cambiar la educación. Madrid, España: Ediciones Morata, 2018. p. 9-45.

GIROUX, Henry. Hacia una pedagogía de la esperanza educada bajo el capitalismo de casino. Pedagogía y Saberes. v. 50, p. 153-158, 2019. Disponível em http://revistas.pedagogica.edu.co/index.php/PYS/article/view/9508/7044. Acceso em 3 nov. 2019.

HELD, Virginia. The Ethics of Care: Personal, Political, and Global. New York: Oxford University Press, 2005.

HERNANDO, Almudena. La fantasía de la individualidad. Madrid: Katz Editores, 2012. 
HERRERO, Yayo. Sujetos arraigados en la tierra y en los cuerpos. Hacia una antropología que reconozca los límites y la vulnerabilidad. In: HERRERO, Yayo; RIECHMANN, Jorge; SANTIAGO, Emilio. (Ed.). Petroleo. Barcelona, España: Arcadia Ediciones, 2018. p. 78-112.

HERRERO, Yayo. Educar para la transformación ecosocial. Disponível em https://www.youtube.com/watch?v=jvYGZ6JLNf0. Acceso em 1 de ago. 2019.

KOLHBERG, Lawrence. Stages in Moral Developmental as a Basis for Moral Education. In Moral Education: Interdiscipplinary Approaches. C.M. Beck, B.S: Cisttenden, E.V. Sullivan. Toronto: Toronto University Press, 1971.

MACEIRA, Luz. Más allá de la coeducación: Pedagogía feminista. Revista educar, v. 36, pp. 27-36, 2006.

MARTÍNEZ-MARTÍN, Irene. Construcción de una pedagogía feminista para una ciudadanía transformadora y contra-hegemónica. Foro de Educación, v. 14, n. 20, pp. 129-151, 2016. Disponível em http://dx.doi.org/10.14516/fde.2016.014.020.008. Acceso em: 10 de sep. 2019.

MARTÍNEZ-MARTÍN, Irene. Pedagogías feministas: estrategias para una educación emancipadora y decolonial. Diálogos em Educaçao. v. 27, n. 3, 2018. Disponível em https://doi.org/10.14295/momento.v27i3.8687. Acceso em: 11 de nov. 2019

MARTÍNEZ-MARTÍN, Irene y RAMÍREZ-ARTIAGA. Des-patriarcalizar y descolonizar la educación: experiencias para una formación feminista del profesorado. Revista Internacional de Educación para la Justicia Social (RIEJS), v. 6, n. 2, 2017. Disponível em 10.15366/riejs2017.6.2.005. Acceso em: 20 de nov. 2019.

NODDINGS, Nel. The Challenge to Care in Schools: An Alternative Approach to Education. Advances in Contemporary Educational Thought, Volume 8. New York: Teachers College Press, 1992.

NODDINGS, Nel. An Ethic of Caring and Its Implications for Instructional Arrangemens. In: THAYER-BACON, Barbara; STONE, Lynda; SPRECHER, Katharine. (Ed.). Education Feminism: Classic and contemporary readings. New York: Suny, dec 2013. p. 137-149.

RUDDICK, Sara. Care as Labor and Relationship. In: RITZER, Jorge. (ed). Encyclopedia of Social Theory. Lanham, MD: Rowman \& Littlefield, 1998. Disponível em: http://dx.doi.org/10.4135/9781412952552.n250. Acceso em 10 ago. 2019.

SÁNCHEZ-SAINZ, Mercedes. Pedagogías Queer: ¿nos arriesgamos a hacer otra educación? Madrid: Catarata, 2019.

SKELTON, C. y FRANCIS, B. Feminism and "The Schooling Scandal". Oxon: Routledge, 2009. 
SUBIRATS, Marina. De los dispositivos selectivos en la educación: el caso del sexismo. Revista de Sociología de la Educación. v. 9, n. 1, p. 22-36, 2016. Disponível em https://ojs.uv.es/index.php/RASE/article/view/8401. Acesso em 28 jun 2018.

TRONTO, Joan C. Creating Caring Institutions: Politics, Plurality, and Purpose. Ethics and Social Welfare. v. 4, n. 2, p. 158-171, 2010. Disponível em https://doi.org/10.1080/17496535.2010.484259. Acceso em: 15 sept. 2019.

TRONTO, Joan C. y FISHER, Berenice. Toward a Feminist Theory of Caring. In: ABEL, Emily y NELSON, Margaret (Ed). Circles of Care. NY: Suny Press, 1990. p. 36-54.

ZANDER, Mary Jane. Becoming Dialogical: Creating a Place for Dialogue. In: Art Education. v. 57, n. 3, p. 48-53, 2004. Disponível em https://doi.org/10.1080/00043125.2004.11653551. Acesso em 1 ago 2019.

UNESCO. Replantear la educación: ¿Hacia un bien común mundial? París: Ediciones UNESCO. 2015. Disponível em https://unesdoc.unesco.org/ark:/48223/pf0000232697. Acesso em 29 out. 2019 